\title{
Existence of Three Positive Solutions for a Class of Boundary Value Problems of Caputo Fractional $q$-Difference Equation
}

\author{
Huiqin Chen, Shugui Kang $\mathbb{D}$, Lili Kong, and Ying Gao \\ School of Mathematics and Computer Sciences, Shanxi Datong University, Datong, Shanxi 037009, China \\ Correspondence should be addressed to Shugui Kang; dtkangshugui@126.com
}

Received 28 November 2017; Revised 9 January 2018; Accepted 28 January 2018; Published 26 February 2018

Academic Editor: Douglas R. Anderson

Copyright (C) 2018 Huiqin Chen et al. This is an open access article distributed under the Creative Commons Attribution License, which permits unrestricted use, distribution, and reproduction in any medium, provided the original work is properly cited.

A class of boundary value problems of Caputo fractional $q$-difference equation is introduced. Green's function and its properties for this problem are deduced. By applying these properties and the Leggett-Williams fixed-point theorem, existence criteria of three positive solutions are obtained. At last, some examples are given to illustrate the validity of our main results.

\section{Introduction}

Some researchers have paid close attention to the research of $q$-difference equation since the $q$-difference calculus and quantum calculus were discovered by Jackson $[1,2]$. After the fractional $q$-difference calculus was developed by Al-Salam et al. [3-6], many papers on the fractional $q$-difference equation kept emerging, such as the papers [7-21] and their references. Among them, Li and Yang [7] established the existence of positive solutions for a class of nonlinear fractional $q$ difference equations with integral boundary conditions by applying monotone iterative method. Koca [8] provided an analytical method that can be used to solve analytically the Caputo fractional $q$-differential equations with initial condition $x(0)=x_{0}$. The advantage of the method is that it can be applied to the integer order $q$-difference equations. By applying the monotone iterative technique combined with the method of lower and upper solutions, Wang et al. [9] obtained the existence of extremal solutions for fractional $q$ difference equation with initial value problem.

There are also many papers about boundary value problems of fractional $q$-difference equations; see [10-19] and the references therein. These experts did researches about the existence of a positive solution and multiple positive solutions to this problem by applying some well-known fixed-point theories such as Krasnosel'skii and Schauder fixed-point theorems. Thereinto, in $[7,15-20]$, the authors focused on the fractional $q$-difference equation with integral boundary value conditions.

Motivated by the methods of [21-23] and the above works, we study the criteria of three positive solutions for a Caputo fractional $q$-difference equation with integral boundary value conditions by employing properties of Green's function and the Leggett-Williams fixed-point theorem in this paper. We mainly consider the following problem:

$$
\begin{aligned}
\left({ }^{C} D_{q}^{v} x\right)(t)+f(t, x(t)) & =0, \\
0 & <q<1,0<t<1,2<v<3, \\
x(0) & =D_{q}^{2} x(0)=0, \\
x(1) & =\lambda \int_{0}^{1} x(s) \mathrm{d}_{q} s,
\end{aligned}
$$

where ${ }^{C} D_{q}^{\nu}$ denotes the Caputo fractional $q$-derivative of order $v$ and $f \in C([0,1] \times[0,+\infty)) \rightarrow[0,+\infty)$ is continuous function.

In Section 2, basic definitions and some lemmas that will be used in the latter part are presented. In Section 3, some results for the existence of three positive solutions to problem (1)-(2) are established. And some examples to corroborate our results are given in Section 4. 


\section{Background Materials and Preliminaries}

In this piece, we show some basic definitions and some lemmas that will be used to demonstrate our main results in the latter section.

Setting $0<q<1$, we define

$$
[\nu]_{q}=\frac{1-q^{\nu}}{1-q}, \quad \nu \in \mathbb{R} .
$$

The $q$-analogue of the power function $(t-s)^{n}$ with $n \in \mathbb{N}$ is

$$
\begin{aligned}
& (t-s)^{0}=1, \\
& (t-s)^{n}=\prod_{k=0}^{n-1}\left(t-s q^{k}\right), \quad n \in \mathbb{N}, t, s \in \mathbb{R} .
\end{aligned}
$$

If $\alpha \in \mathbb{R}$, then

$$
(t-s)^{(\alpha)}=t^{\alpha} \prod_{n=0}^{\infty} \frac{t-s q^{n}}{t-s q^{n+\alpha}}
$$

We define the $q$-gamma function as follows:

$$
\begin{aligned}
\Gamma_{q}(s)=(1-q)^{(s-1)}(1-q)^{1-s}, & \\
& \\
& s \in \mathbb{R} \backslash\{0,-1,-2, \ldots\},
\end{aligned}
$$

which satisfies $\Gamma_{q}(s+1)=[s]_{q} \Gamma_{q}(s)$. by

For $0<q<1$, the $q$-derivative of a function $f$ is defined

$$
\begin{aligned}
& \left(D_{q} f\right)(t)=\frac{\mathrm{d}_{q}}{\mathrm{~d}_{q} t} f(t)=\frac{f(t)-f(q t)}{(1-q) t}, \\
& \left(D_{q} f\right)(0)=\lim _{t \rightarrow 0}\left(D_{q} f\right)(t),
\end{aligned}
$$

$$
t \neq 0 \text {. }
$$

The higher order $q$-derivatives are defined by

$$
\begin{aligned}
& \left(D_{q}^{0} f\right)(t)=f(t), \\
& \left(D_{q}^{n} f\right)(t)=D_{q}\left(D_{q}^{n-1} f\right)(t), \quad n \in \mathbb{N} .
\end{aligned}
$$

The $q$-integral of a function $f$ defined on the interval $[0, b]$ is given by

$$
\left(I_{q} f\right)(t)=\int_{0}^{t} f(s) \mathrm{d}_{q} s=t(1-q) \sum_{n=0}^{\infty} f\left(t q^{n}\right) q^{n}
$$

$$
t \in[0, b]
$$

If $f$ is defined on the interval $[0, b]$ and $a \in[0, b]$, its $q$ integral from $a$ to $b$ is defined by

$$
\int_{a}^{b} f(s) \mathrm{d}_{q} s=\int_{0}^{b} f(s) \mathrm{d}_{q} s-\int_{0}^{a} f(s) \mathrm{d}_{q} s .
$$

The higher order $q$-integrals are defined by

$$
\begin{aligned}
& \left(I_{q}^{0} f\right)(t)=f(t) \\
& \left(I_{q}^{n} f\right)(t)=I_{q}\left(I_{q}^{n-1} f\right)(t), \quad n \in \mathbb{N} .
\end{aligned}
$$

We note that $\left(D_{q} I_{q} f\right)(t)=f(t)$ and if $f$ is continuous at $x=0$, we get $\left(I_{q} D_{q} f\right)(t)=f(t)-f(0)$. For more details on the basic material of $q$-calculus, the readers can refer to [1-6].

Now let us give definitions of fractional $q$-integral and $q$ derivative.

Definition 1 (see $[4,6]$ ). Let $v \geqslant 0$ and let $f$ be a function defined on $L^{1}([0,1])$. The fractional $q$-integral of the Riemann-Liouville type is $\left(I_{q}^{0} f\right)(t)=f(t)$ and

$$
\begin{aligned}
\left(I_{q}^{v} f\right)(t)=\frac{1}{\Gamma_{q}(\nu)} \int_{0}^{t}(t-q s)^{(\nu-1)} f(s) \mathrm{d}_{q} s, & \\
& v>0, t \in[0,1],
\end{aligned}
$$

where $L^{1}([0,1])$ denotes the classical Banach space consisting of measurable functions on $[0,1]$ that are integrable.

Definition 2 (see [6]). The fractional $q$-derivative of the Riemann-Liouville type of order $v \geqslant 0$ is given by $D_{q}^{0} f(t)=$ $f(t)$ and

$$
\left(D_{q}^{v} f\right)(t)=\left(D_{q}^{n} I_{q}^{n-v} f\right)(t)
$$

where $n$ is the smallest integer greater than or equal to $v$.

Definition 3 (see [13]). The fractional $q$-derivative of Caputo type of order $v \geqslant 0$ for a function $f$ is defined by

$$
\left({ }^{C} D_{q}^{v} f\right)(t)=\left(I_{q}^{n-v} D_{q}^{n} f\right)(t)
$$


Lemma 4 (see [13]). Let $\nu>0$ and let $n$ be the smallest integer greater than or equal to $v$. Then, for $t \in[0,1]$, the following equality holds:

$$
\left(I_{q}^{\nu C} D_{q}^{\nu} f\right)(t)=f(t)+\sum_{k=0}^{n-1} \frac{t^{k}}{\Gamma_{q}(k+1)} D_{q}^{k} f(0) .
$$

Lemma 5 (see [13]). Let $\alpha \in \mathbb{R}_{+}, \beta \in(-1,+\infty)$, and the following is valid:

$$
I_{q}^{\alpha}(t-s)^{(\beta)}=\frac{\Gamma_{q}(\beta+1)}{\Gamma_{q}(\beta+\alpha+1)}(t-s)^{(\beta+\alpha)} .
$$

Next, Green's function for integral boundary value problem (1)-(2) is derived and the properties of Green's function are concluded. These properties will be used to demonstrate the main results in Section 3.
Lemma 6. Given $g(t) \in C[0,1]$, the unique solution of the following problem

$$
\begin{aligned}
\left({ }^{C} D_{q}^{v} x\right)(t)+g(t) & =0, \quad 0<t<1,2<v<3, \\
x(0) & =D_{q}^{2} x(0)=0, \\
x(1) & =\lambda \int_{0}^{1} x(s) \mathrm{d}_{q} s
\end{aligned}
$$

is

$$
x(t)=\int_{0}^{1} G(t, q s) g(s) \mathrm{d}_{q} s
$$

where

$$
G(t, q s)=\frac{1}{\tau \Gamma_{q}(\nu)} \begin{cases}{[2]_{q} t(1-q s)^{(\nu-1)}\left([\nu]_{q}-\lambda+\lambda s q^{\nu}\right)-\tau(t-q s)^{(\nu-1)},} & 0 \leqslant q s \leqslant t \leqslant 1 \\ {[2]_{q} t(1-q s)^{(\nu-1)}\left([\nu]_{q}-\lambda+\lambda s q^{\nu}\right),} & 0 \leqslant t \leqslant q s \leqslant 1\end{cases}
$$

and $\tau=\left([2]_{q}-\lambda\right)[\nu]_{\mathrm{q}}$. Here $G(t, q s)$ is called Green's function of boundary value problem (17)-(18).

Proof. By Lemma 4, it is clear that (17) is equivalent to

$$
\begin{aligned}
x(t)= & -\frac{1}{\Gamma_{q}(\nu)} \int_{0}^{t}(t-q s)^{(\nu-1)} g(s) \mathrm{d}_{q} s+c_{1}+c_{2} t \\
& +c_{3} t^{2}
\end{aligned}
$$

for some $c_{i} \in \mathbb{R}, i=1,2,3$. Applying the boundary condition $x(0)=D_{q}^{2} x(0)=0$, there is $c_{1}=c_{3}=0$, and then

$$
x(t)=-\frac{1}{\Gamma_{q}(\nu)} \int_{0}^{t}(t-q s)^{(\nu-1)} g(s) \mathrm{d}_{q} s+c_{2} t .
$$

Using the condition $x(1)=\lambda \int_{0}^{1} x(s) \mathrm{d}_{q} s$, there is

$$
c_{2}=\frac{1}{\Gamma_{q}(\nu)} \int_{0}^{1}(1-q s)^{(\nu-1)} g(s) \mathrm{d}_{q} s+\lambda \int_{0}^{1} x(s) \mathrm{d}_{q} s .
$$

Substituting $c_{2}$ into (22), we get

$$
\begin{aligned}
x(t)= & -\frac{1}{\Gamma_{q}(\nu)} \int_{0}^{t}(t-q s)^{(\nu-1)} g(s) \mathrm{d}_{q} s \\
& +\frac{t}{\Gamma_{q}(\nu)} \int_{0}^{1}(1-q s)^{(\nu-1)} g(s) \mathrm{d}_{q} s \\
& +\lambda t \int_{0}^{1} x(s) \mathrm{d}_{q} s .
\end{aligned}
$$

Let $\xi=\int_{0}^{1} x(s) \mathrm{d}_{q} s$; integrating equality (24) with respect to $t$ from $t=0$ to $t=1$ and then exchanging integral order, we obtain that

$$
\begin{aligned}
\xi= & -\frac{1}{\Gamma_{q}(\nu)} \int_{0}^{1} \mathrm{~d}_{q} t \int_{0}^{t}(t-q s)^{(\nu-1)} g(s) \mathrm{d}_{q} s \\
& +\frac{1}{\Gamma_{q}(\nu)} \int_{0}^{1} t \mathrm{~d}_{q} t \int_{0}^{1}(1-q s)^{(\nu-1)} g(s) \mathrm{d}_{q} s \\
& +\lambda \xi \int_{0}^{1} s \mathrm{~d}_{q} s \\
= & -\frac{1}{[\nu]_{q} \Gamma_{q}(\nu)} \int_{0}^{1}(1-q s)^{(\nu)} g(s) \mathrm{d}_{q} s \\
& +\frac{1}{[2]_{q} \Gamma_{q}(\nu)} \int_{0}^{1}(1-q s)^{(\nu-1)} g(s) \mathrm{d}_{q} s+\frac{\lambda \xi}{[2]_{q}} .
\end{aligned}
$$

Solving the above equation, then

$$
\begin{aligned}
\xi= & -\frac{[2]_{q}}{\tau \Gamma_{q}(\nu)} \int_{0}^{1}(1-q s)^{(\nu)} g(s) \mathrm{d}_{q} s \\
& +\frac{[\nu]_{q}}{\tau \Gamma_{q}(\nu)} \int_{0}^{1}(1-q s)^{(\nu-1)} g(s) \mathrm{d}_{q} s .
\end{aligned}
$$

Substituting $\xi$ into (24), we get

$$
\begin{gathered}
x(t)=-\frac{1}{\Gamma_{q}(\nu)} \int_{0}^{t}(t-q s)^{(\nu-1)} g(s) \mathrm{d}_{q} s+\frac{1}{\tau \Gamma_{q}(\nu)} \\
\cdot \int_{0}^{1}[2]_{q} t(1-q s)^{(\nu-1)}\left([\nu]_{q}-\lambda+\lambda s q^{\nu}\right)
\end{gathered}
$$




$$
\begin{aligned}
& \cdot g(s) \mathrm{d}_{q} s=\frac{1}{\tau \Gamma_{q}(\nu)} \\
& \cdot \int_{0}^{t}\left\{[2]_{q} t(1-q s)^{(\nu-1)}\left([\nu]_{q}-\lambda+\lambda s q^{\nu}\right)\right. \\
& \left.-\tau(t-q s)^{(\nu-1)}\right\} g(s) \mathrm{d}_{q} s+\frac{1}{\tau \Gamma_{q}(\nu)} \int_{t}^{1}[2]_{q} t(1 \\
& -q s)^{(\nu-1)}\left([\nu]_{q}-\lambda+\lambda s q^{\nu}\right) g(s) \mathrm{d}_{q} s \\
& =\int_{0}^{1} G(t, q s) g(s) \mathrm{d}_{q} s .
\end{aligned}
$$

Remark 7. It is obvious that $G(0, q s)=G(t, 1)=0$ for all $t, q s \in[0,1]$ and $\lambda \neq[2]_{q}$.

Lemma 8. Suppose $2<v<3$ and $0<\lambda<[2]_{q}$. Then the function $G(t, q s)$ defined by (20) satisfies the following inequalities:

$$
t G(1, q s) \leqslant G(t, q s) \leqslant \frac{[2]_{q}[\nu]_{q}}{\lambda\left([\nu]_{q}-[2]_{q}\right)} G(1, q s)
$$

The proof is complete. $(t, q s) \in[0,1] \times[0,1]$.

Proof. According to the expression of $G(t, q s)$, we get

$$
\frac{G(t, q s)}{G(1, q s)}= \begin{cases}\frac{[2]_{q} t(1-q s)^{(\nu-1)}\left([\nu]_{q}-\lambda+\lambda s q^{\nu}\right)-\tau(t-q s)^{(\nu-1)}}{(1-q s)^{(\nu-1)} \lambda\left([\nu]_{q}-[2]_{q}\left(1-s q^{\nu}\right)\right)}, & 0 \leqslant q s \leqslant t \leqslant 1 \\ \frac{[2]_{q} t\left([\nu]_{q}-\lambda+\lambda s q^{\nu}\right)}{\lambda\left([\nu]_{q}-[2]_{q}\left(1-s q^{\nu}\right)\right)}, & 0 \leqslant t \leqslant q s \leqslant 1 .\end{cases}
$$

For the case $0 \leqslant t \leqslant q s \leqslant 1$, it is clear that

$$
\begin{aligned}
\frac{G(t, q s)}{G(1, q s)} & \leqslant \frac{[2]_{q}[\nu]_{q}}{\lambda\left([\nu]_{q}-[2]_{q}\right)} \\
\frac{G(t, q s)}{G(1, q s)} & \geqslant \frac{[2]_{q} t\left([2]_{q}\left(s q^{\nu}-1\right)+[\nu]_{q}\right)}{\lambda\left([2]_{q}\left(s q^{\nu}-1\right)+[\nu]_{q}\right)}=\frac{[2]_{q} t}{\lambda} \\
& \geqslant t
\end{aligned}
$$

For the case $0 \leqslant q s \leqslant t \leqslant 1$, it is easy to see that

$$
\begin{aligned}
\frac{G(t, q s)}{G(1, q s)} & \leqslant \frac{[2]_{q} t\left([\nu]_{q}-\lambda+\lambda s q^{\nu}\right)}{\lambda\left([\nu]_{q}-[2]_{q}\left(1-s q^{\nu}\right)\right)} \\
& \leqslant \frac{[2]_{q}[\nu]_{q}}{\lambda\left([\nu]_{q}-[2]_{q}\right)} .
\end{aligned}
$$

On the other hand,

$$
\begin{aligned}
& \frac{G(t, q s)}{G(1, q s)} \\
& \geqslant \frac{[2]_{q} t(1-q s)^{(\nu-1)}\left([\nu]_{q}-\lambda+\lambda s q^{\nu}\right)-\tau(t-t q s)^{(\nu-1)}}{(1-q s)^{(\nu-1)} \lambda\left([\nu]_{q}-[2]_{q}\left(1-s q^{\nu}\right)\right)} \\
& =\frac{[2]_{q} t\left([\nu]_{q}-\lambda+\lambda s q^{\nu}\right)-\left([2]_{q}-\lambda\right)[\nu]_{q} t^{\nu-1}}{\lambda\left([\nu]_{q}-[2]_{q}\left(1-s q^{\nu}\right)\right)}
\end{aligned}
$$

$$
\begin{aligned}
& \geqslant \frac{[2]_{q} t\left([\nu]_{q}-\lambda+\lambda s q^{\nu}\right)-\left([2]_{q}-\lambda\right)[\nu]_{q} t}{\lambda\left([\nu]_{q}-[2]_{q}\left(1-s q^{\nu}\right)\right)} \\
& =\frac{t\left(-[2]_{q} \lambda+[2]_{q} \lambda s q^{\nu}+\lambda[\nu]_{q}\right)}{\lambda\left([\nu]_{q}-[2]_{q}\left(1-s q^{\nu}\right)\right)}=t .
\end{aligned}
$$

Therefore

$$
\begin{aligned}
t G(1, q s) \leqslant G(t, q s) \leqslant \frac{[2]_{q}[\nu]_{q}}{\lambda\left([\nu]_{q}-[2]_{q}\right)} G(1, q s) & \\
& 0 \leqslant q s<t \leqslant 1 .
\end{aligned}
$$

When $t=1$, inequalities (28) are obvious. In conclusion, inequalities (28) are fulfilled. The proof is complete.

Corollary 9. If $[\nu]_{q}-[2]_{q}>0$, then $G(t, q s)>0$, for $t, q s \in$ $(0,1)$.

Definition 10 (see [24]). If $P$ is a cone of the real Banach space $E$, a mapping $\theta: P \rightarrow[0, \infty)$ is continuous and with

$$
\begin{array}{r}
\theta(t x+(1-t) y) \geqslant t \theta(x)+(1-t) \theta(y), \\
x, y \in P, t \in[0,1],
\end{array}
$$

it is called a nonnegative concave continuous functional $\theta$ on $P$. 
Assuming that $r, a, b$ are positive constants, we will employ the following notations:

$$
\begin{aligned}
P_{r} & =\{x \in P:\|x\|<r\}, \\
\bar{P}_{r} & =\{x \in P:\|x\| \leqslant r\}, \\
P(\theta, a, b) & =\{x \in P: \theta(x) \geqslant a,\|x\| \leqslant b\} .
\end{aligned}
$$

Our existence criteria will be based on the following Leggett-Williams fixed-point theorem.

Lemma 11 (see [24]). Let $E=(E,\|\cdot\|)$ be a Banach space, $P \subset E$ be a cone of $E$, and $c>0$ be a constant. Suppose there exists a concave nonnegative continuous functional $\theta$ on $P$ with $\theta(x) \leqslant\|x\|$ for $x \in \bar{P}_{c}$. Let $T: \bar{P}_{c} \rightarrow \bar{P}_{c}$ be a completely continuous operator. Assume there are numbers, $a$, and $b$ with $0<d<a<b \leq c$ such that

(i) the set $\{x \in P(\theta, a, b): \theta(x)>a\}$ is nonempty and $\theta(T x)>a$ for all $x \in P(\theta, a, b)$;

(ii) $\|T x\|<d$ for $x \in \bar{P}_{d}$;

(iii) $\theta(T x)>a$ for all $x \in P(\theta, a, c)$ with $\|T x\|>b$.

Then $T$ has at least three fixed points $x_{1}, x_{2}$, and $x_{3} \in \bar{P}_{c}$. Furthermore, we have

$$
\begin{aligned}
\max _{t \in[0,1]} x_{1}(t) & <d, \\
a & <\min _{t \in[0,1]} x_{2}(t)<\max _{t \in[0,1]} x_{2}(t)<c, \\
d & <\max _{t \in[0,1]} x_{3}(t) \leq c, \\
\min _{t \in[0,1]} x_{3}(t) & <a .
\end{aligned}
$$

\section{Existence of Three Positive Solutions}

In this section, the above lemmas will be applied to obtain the main results of this paper.

Let $C[0,1]$ be the space of all continuous real functions defined on $[0,1]$ with the maximum norm $\|x\|=$ $\max _{t \in[0,1]}|x(t)|$. We can know it is a Banach space. Define the cone $P \subset C[0,1]$ as follows:

$$
P=\{x \in C[0,1]: x(t) \geqslant 0, t \in[0,1]\} .
$$

From Lemma 6, we know that $x(t)$ is a solution of boundary value problem (1)-(2) if and only if it satisfies

$$
x(t)=\int_{0}^{1} G(t, q s) f(s, x(s)) \mathrm{d}_{q} s, \quad t \in[0,1] .
$$

That is to say, the positive solutions of problem (1)-(2) are equivalent to the fixed points of $T$ in $C[0,1]$ defined by

$$
(T x)(t)=\int_{0}^{1} G(t, q s) f(s, x(s)) \mathrm{d}_{q} s, \quad t \in[0,1] .
$$

Then $T(P) \subset P$ and using the Ascoli-Arzelà theorem, we are able to confirm that $T$ is completely continuous.
We shall use Lemma 11 to discuss the existence of three fixed points to $T$. We then obtain sufficient conditions for the existence of three positive solutions to problem (1)-(2). To establish our main results, we take a positive number $\mu \epsilon$ $(0,1)$, letting the nonnegative concave continuous function $\theta$ on $P$ be defined by

$$
\theta(x)=\min _{t \in[\mu, 1]} x(t)
$$

Denote

$$
\begin{aligned}
f^{0} & =\lim _{x \rightarrow 0} \sup _{t \in[0,1]} \frac{f(t, x)}{x} \\
f^{\infty} & =\lim _{x \rightarrow+\infty} \sup _{t \in[0,1]} \frac{f(t, x)}{x} \\
A^{-1} & =\int_{0}^{1} \frac{[2]_{q}[\nu]_{q}}{\lambda\left([\nu]_{q}-[2]_{q}\right)} G(1, q s) \mathrm{d}_{q} s, \\
B^{-1} & =\int_{\mu}^{1} G(1, q s) \mathrm{d}_{q} s \\
\rho & =\frac{[\nu]_{q}[2]_{q}}{\lambda \mu\left([\nu]_{q}-[2]_{q}\right)} .
\end{aligned}
$$

And suppose that the function $f(t, x)$ satisfies the following condition:

(C) $f(t, x)$ is a nonnegative continuous function on $[0,1] \times[0,+\infty)$ and there exists $t_{n} \rightarrow 0$ such that $f\left(t_{n}, x\left(t_{n}\right)\right)>0, n=1,2, \ldots$.

Theorem 12. Assume that condition (C) holds and there exist constants $0<d<a$ such that

(C1) $f(t, x)<$ Ad for $(t, x) \in[0,1] \times[0, d]$;

(C2) $f(t, x) \geqslant(B / \mu)$ a for $(t, x) \in[\mu, 1] \times[a, c]$, where $c>$ $\rho a$;

(C3) $f(t, x) \leqslant \kappa x+\beta$ for $(t, x) \in[0,1] \times[0,+\infty)$, where $\kappa, \beta$ are positive numbers.

Then the boundary value problem (1)-(2) has at least three positive solutions $x_{1}, x_{2}$, and $x_{3}$.

Proof. Set $c>\max \{\beta /(A-\kappa), \rho a\}$, and then, for $x \in \bar{P}_{c}$, we have from (28)

$$
\begin{aligned}
\|T x\| & =\max _{t \in[0,1]} \int_{0}^{1} G(t, q s) f(s, x(s)) \mathrm{d}_{q} s \\
& \leqslant \int_{0}^{1} \frac{[2]_{q}[\nu]_{q}}{\lambda\left([\nu]_{q}-[2]_{q}\right)} G(1, q s)(\kappa x(s)+\beta) \mathrm{d}_{q} s \\
& \leqslant(\kappa\|x\|+\beta) \int_{0}^{1} \frac{[2]_{q}[\nu]_{q}}{\lambda\left([\nu]_{q}-[2]_{q}\right)} G(1, q s) \mathrm{d}_{q} s \\
& =\frac{(\kappa\|x\|+\beta)}{A}<c .
\end{aligned}
$$


That is, $T x \in P_{c}$. Therefore $T: \bar{P}_{c} \rightarrow \bar{P}_{c}$ is a completely continuous operator. By $(\mathrm{C} 1)$, we can get

$$
\begin{aligned}
\|T x\| & \leqslant \int_{0}^{1} \frac{[2]_{q}[\nu]_{q}}{\lambda\left([\nu]_{q}-[2]_{q}\right)} G(1, q s) f(s, x(s)) \mathrm{d}_{q} s \\
& <A d \int_{0}^{1} \frac{[2]_{q}[\nu]_{q}}{\lambda\left([\nu]_{q}-[2]_{q}\right)} G(1, q s) \mathrm{d}_{q} s=d .
\end{aligned}
$$

Hence condition (ii) of Lemma 11 is satisfied.

We choose $x_{0}=(\rho+1) a / 2$ for $t \in[\mu, 1]$; then $x_{0} \in$ $\{x \in P(\theta, a, \rho a): \theta(x)>a\}$, which implies $\{x \in P(\theta, a, \rho a)$ : $\theta(x)>a\} \neq \emptyset$. Hence, if $x \in P(\theta, a, \rho a)$, then $a \leqslant x(t) \leqslant \rho a$ for $\mu \leqslant t \leqslant 1$. Thus

$$
\begin{aligned}
\theta(T x) & =\min _{\mu \leqslant t \leqslant 1} \int_{0}^{1} G(t, q s) f(s, x(s)) \mathrm{d}_{q} s \\
& >\int_{\mu}^{1} \min _{\mu \leqslant t \leqslant 1} G(t, q s) f(s, x(s)) \mathrm{d}_{q} s \\
& >\int_{\mu}^{1} \mu G(1, q s) f(s, x(s)) \mathrm{d}_{q} s \\
& \geqslant \frac{B}{\mu} a \int_{\mu}^{1} \mu G(1, q s) \mathrm{d}_{q} s=a .
\end{aligned}
$$

From the above inequality, we see that $\theta(T x)>a$ for all $x \in P(\theta, a, \rho a)$. This affirms that condition (i) of Lemma 11 is satisfied.

Finally, for $x \in P(\theta, a, c)$ with $\|T x\|>\rho a$, we get

$$
\begin{aligned}
\theta(T x) & =\min _{\mu \leqslant t \leqslant 1} \int_{0}^{1} G(t, q s) f(s, x(s)) \mathrm{d}_{q} s \\
& \geqslant \mu \int_{0}^{1} G(1, q s) f(s, x(s)) \mathrm{d}_{q} s \\
& =\frac{1}{\rho} \int_{0}^{1} \frac{[\nu]_{q}[2]_{q}}{\lambda\left([\nu]_{q}-[2]_{q}\right)} G(1, q s) f(s, x(s)) \mathrm{d}_{q} s \\
& \geqslant \frac{1}{\rho} \max _{0 \leqslant t \leqslant 1} \int_{0}^{1} G(t, q s) f(s, x(s)) \mathrm{d}_{q} s>\frac{1}{\rho} \cdot \rho a \\
& =a .
\end{aligned}
$$

This confirms that condition (iii) of Lemma 11 is fulfilled. By virtue of Lemma 11, the boundary value problem (1)-(2) has at least three solutions $x_{1}, x_{2}$, and $x_{3}$. Taking into account the fact that condition (C) holds, we have $x_{i}(t)>0,0<t<$ $1, i=1,2,3$. The proof is complete.

Theorem 13. Let condition (C) hold. Assume that there exist constants $0<d<a<c(c>\rho a)$ such that (C1), (C2), and (C4) are satisfied, where

(C4) $f(t, x) \leqslant$ Ac for $(t, x) \in[0,1] \times[0, c]$.
Then the boundary value problem (1)-(2) has at least three positive solutions $x_{1}, x_{2}$, and $x_{3}$ such that

$$
\begin{aligned}
\max _{t \in[0,1]} x_{1}(t) & <d, \\
a & <\min _{t \in[\mu, 1]} x_{2}(t)<\max _{t \in[0,1]} x_{2}(t)<c, \\
d & <\max _{t \in[0,1]} x_{3}(t) \leqslant c, \\
\min _{t \in[\mu, 1]} x_{3}(t) & <a .
\end{aligned}
$$

Proof. From (C4), we get

$$
\begin{aligned}
\|T x\| & =\max _{t \in[0,1]} \int_{0}^{1} G(t, q s) f(s, x(s)) \mathrm{d}_{q} s \\
& \leqslant \int_{0}^{1} \frac{[2]_{q}[\nu]_{q}}{\lambda\left([\nu]_{q}-[2]_{q}\right)} G(1, q s) f(s, x(s)) \mathrm{d}_{q} s \\
& <A c \int_{0}^{1} \frac{[2]_{q}[\nu]_{q}}{\lambda\left([\nu]_{q}-[2]_{q}\right)} G(1, q s) \mathrm{d}_{q} s=c .
\end{aligned}
$$

Therefore, $T: \bar{P}_{c} \rightarrow \bar{P}_{c}$. The remainder of the proof is similar to the proof of Theorem 12 and is therefore omitted. By Lemma 11, the boundary value problem (1)-(2) has at least three positive solutions $x_{1}, x_{2}$, and $x_{3}$ satisfying

$$
\begin{aligned}
\max _{t \in[0,1]} x_{1}(t) & <d, \\
a & <\min _{t \in[\mu, 1]} x_{2}(t)<\max _{t \in[0,1]} x_{2}(t)<c, \\
d & <\max _{t \in[0,1]} x_{3}(t) \leqslant c, \\
\min _{t \in[\mu, 1]} x_{3}(t) & <a .
\end{aligned}
$$

The proof is complete.

Theorem 14. Let condition $(C)$ hold. Assume that there exist constants $0<d<$ a such that (C1) and (C2) are satisfied, and function $f(t, s)$ satisfies

$$
\text { (C5) } f^{\infty}<A \text {. }
$$

Then the boundary vale problem (1)-(2) has at least three positive solutions.

Proof. From hypothesis (C5), there exist $0<\sigma<A$ and $R>$ 0 ; when $x \geqslant R$, we have

$$
f(t, x) \leqslant \sigma u
$$

Set $M=\max _{(t, x) \in[0,1] \times[0, R]} f(t, x)$; consequently we get

$$
0 \leqslant f(t, x) \leqslant \sigma x+M, \quad 0 \leqslant x<+\infty .
$$

This shows that condition (C3) of Theorem 12 is satisfied. By Theorem 12, the boundary value problem (1)-(2) has at least three positive solutions. The proof is complete. 
Theorem 15. Assume that there exist two positive constants $a, c(c>\rho a)$ such that conditions (C), (C2), and (C4) hold. And function $f(t, x)$ satisfies

(C6) $f^{0}<A$.

Then the boundary value problem (1)-(2) has at least three positive solutions.

Proof. In line with (C6), it is easy to see that there exists a positive constant $d<a$ such that, for $\|x\|<d$, we have

$$
f(t, x(t))<A x .
$$

That is to say,

$$
f(t, x(t))<A d, \quad\|x\|<d .
$$

This implies that conditions of Theorem 13 are satisfied. By Theorem 13, the boundary value problem (1)-(2) has at least three positive solutions. The proof is complete.

In light of the proof of Theorems 14 and 15, we obtain one theorem and four corollaries as follows.

Theorem 16. Assume that the function $f(t, x)$ satisfies conditions (C), (C2), (C5), and (C6). Then the boundary value problem (1)-(2) has at least three positive solutions.

Corollary 17. Assume that conditions (C), (C2), and (C3) hold. The function $f(t, x)$ satisfies $f^{0}=0$. Then the boundary value problem (1)-(2) has at least three positive solutions.

Corollary 18. Assume that conditions (C), (C1), and (C2) hold. The function $f(t, x)$ satisfies $f^{\infty}=0$. Then the boundary value problem (1)-(2) has at least three positive solutions.

Corollary 19. Assume that conditions (C), (C2), and (C4) hold. The function $f(t, x)$ satisfies $f^{\infty}=0$. Then the boundary value problem (1)-(2) has at least three positive solutions.

Corollary 20. Assume that conditions (C) and (C2) hold. The function $f(t, x)$ satisfies $f^{0}=0$ and $f^{\infty}=0$. Then the boundary value problem (1)-(2) has at least three positive solutions.

\section{Examples}

In this section, we present three examples to illustrate our results. We take $v=5 / 2, q=1 / 2, \mu=1 / 2, \lambda=1 / 2$, and by estimation, we then have $A>0.65, B<17.785$.

Consider the Caputo fractional $q$-difference

$$
\left({ }^{C} D_{q}^{v} x\right)(t)+f(t, x)=0, \quad 0<t<1,
$$

with the boundary conditions

$$
\begin{aligned}
& x(0)=D_{q}^{2} x(0)=0, \\
& x(1)=\frac{1}{2} \int_{0}^{1} x(s) \mathrm{d}_{q} s .
\end{aligned}
$$

Example 1. We take

$$
\begin{aligned}
& f(t, x) \\
& \quad= \begin{cases}\frac{2 t}{125}+36 x^{3}, & (t, x) \in[0,1] \times[0,1], \\
\frac{2 t}{125}+35+x, & (t, x) \in[0,1] \times[1,+\infty) .\end{cases}
\end{aligned}
$$

There exist constants $d=1 / 36$ and $a=253 / 250$ such that

$$
\begin{aligned}
& f(t, x)=\frac{2 t}{125}+36 x^{3} \leqslant 0.0167716<0.65 \times \frac{1}{36} d \\
&<A d \quad \text { for }(t, x) \in[0,1] \times\left[0, \frac{1}{36}\right] ; \\
& f(t, x)=\frac{2 t}{125}+35+x \geqslant 36.02>17.785 \cdot \frac{1}{\mu} a>\frac{B}{\mu} a \\
& \text { for }(t, x) \in[\mu, 1] \times[a, \rho a+1] ;
\end{aligned}
$$

$f(t, x) \leqslant 2 / 125+36 x$

$$
\text { for }(t, x) \in[0,1] \times[0,+\infty) \text {. }
$$

All the conditions of Theorem 12 hold. Thus, at this moment, by virtue of Theorem 12 we know that the boundary value problem (53)-(54) has three positive solutions.

Example 2. We take

$$
\begin{aligned}
& f(t, x) \\
& = \begin{cases}100 e^{-t} x^{5}, & (t, x) \in[0,1] \times[0,1], \\
e^{-t}\left[99.35 x^{1 / 2}+0.65 x\right], & (t, x) \in[0,1] \times[1,+\infty) .\end{cases}
\end{aligned}
$$

There exist constants $d=0.25$ and $a=1.06$ such that

$$
\begin{aligned}
& f^{\infty}=0.65<A \text { for }(t, x) \in[0,1] \times[0,+\infty) ; \\
& f(t, x) \geqslant 37.8822>17.785 \cdot \frac{1}{\mu} a>\frac{B}{\mu} a \\
& \text { for }(t, x) \in[\mu, 1] \times[1.06, \rho a+1] ; \\
& f(t, x) \leqslant 100 d^{5} \leqslant 100 d
\end{aligned}
$$$$
\text { for }(t, x) \in[0,1] \times[0,0.25] \text {. }
$$

All the conditions of Theorem 14 hold. Thus, in this case, by Theorem 14 we know that the boundary value problem (53)(54) has three positive solutions.

Example 3. We seek

$$
\begin{aligned}
& f(t, x) \\
& \quad= \begin{cases}0.5 x+45.1 x^{3}, & (t, x) \in[0,1] \times[0,1], \\
45 x^{1 / 2}+0.6 x, & (t, x) \in[0,1] \times[1,+\infty) .\end{cases}
\end{aligned}
$$


There exists constant $a=1.5$ such that

$$
\begin{aligned}
f^{\infty} & =0.6<0.65<A ; \\
f^{0} & =0.5<0.65<A \\
f(t, x) & =45 x^{1 / 2}+0.6 x \geqslant 56.0135>17.785 \cdot \frac{1}{\mu} a \\
& >\frac{B}{\mu} a \text { for }(t, x) \in[\mu, 1] \times[a, \rho a+1] .
\end{aligned}
$$

All the conditions of Theorem 16 hold. Thus, in this case, by using Theorem 16 we know that the boundary value problem (53)-(54) has three positive solutions.

\section{Conclusions}

The main innovation of this paper was that existence criteria of three positive solutions for a Caputo fractional $q$-difference equation with integral boundary value conditions are discussed. The study in the paper was to provide an analytical method: The Leggett-Williams fixed-point theorem can be used to solve fractional $q$-difference equation. In order to use the Leggett-Williams fixed-point theorem, Green's function and its properties were derived. By applying these properties and the Leggett-Williams fixed-point theorem, we presented the existence of three positive solutions of this class of fractional $q$-difference equations with integral boundary value conditions. An important advantage of this method is that it can be used to study three positive solutions for integer order $q$-differential equations and fractional differential equation, and so forth.

\section{Conflicts of Interest}

The authors declare that they have no conflicts of interest.

\section{Acknowledgments}

This project is supported by the National Natural Science Foundation of China (Grant no. 11271235), the Development Foundation of Higher Education Department of Shanxi Province (20101109, 20111117, and 20111020), and Shanxi Datong University Institute (2016K9 and 2017K4).

\section{References}

[1] F. Jackson, "On $q$-functions and a certain difference operator," Transactions of the Royal Society of Edinburgh, vol. 46, pp. 253281, 1908.

[2] F. Jackson, "On q-definite integrals," The Quarterly Journal of Pure and Applied Mathematics, vol. 41, pp. 193-203, 1910.

[3] W. A. Al-Salam, "Some fractional $q$-integrals and $q$-derivatives," Proceedings of the Edinburgh Mathematical Society, vol. 15, no. 2, pp. 135-140, 1967.

[4] R. P. Agarwal, "Certain fractional $q$-integrals and q-derivatives," Mathematical Proceedings of the Cambridge Philosophical Society, vol. 66, pp. 365-370, 1969.
[5] F. M. Atici and P. W. Eloe, "Fractional $q$-calculus on a time scale," Journal of Nonlinear Mathematical Physics, vol. 14, no. 3, pp. 333-344, 2007.

[6] P. M. Rajković, S. D. Marinković, and M. S. Stanković, "Fractional integrals and derivatives in q-calculus," Applicable Analysis and Discrete Mathematics, vol. 1, no. 1, pp. 311-323, 2007.

[7] Y. Li and W. Yang, "Monotone iterative method for nonlinear fractional $q$-difference equations with integral boundary conditions," Advances in Difference Equations, vol. 2015, article 294, 2015.

[8] I. Koca, "A method for solving differential equations of $q$ fractional order," Applied Mathematics and Computation, vol. 266, pp. 1-5, 2015.

[9] G. Wang, W. Sudsutad, L. Zhang, and J. Tariboon, "Monotone iterative technique for a nonlinear fractional $q$-difference equation of Caputo type," Advances in Difference Equations, vol. 2016, article no. 211, 2016.

[10] R. A. Ferreira, "Positive solutions for a class of boundary value problems with fractional $q$-differences," Computers \& Mathematics with Applications. An International Journal, vol. 61, no. 2, pp. 367-373, 2011.

[11] X. Li, Z. Han, and S. Sun, "Existence of positive solutions of nonlinear fractional $q$-difference equation with parameter," Advances in Difference Equations, vol. 2013, article 260, 2013.

[12] X. Li, Z. Han, S. Sun, and L. Sun, "Eigenvalue problems of fractional $q$-difference equations with generalized $p$-Laplacian," Applied Mathematics Letters, vol. 57, pp. 46-53, 2016.

[13] B. Ahmad, S. K. Ntouyas, and I. K. Purnaras, "Existence results for nonlocal boundary value problems of nonlinear fractional $q$-difference equations Difference equations: new trends and applications in biology, medicine and biotechnology," Advances in Difference Equations, vol. 2012, article 140, 2012.

[14] W.-X. Zhou and H.-Z. Liu, "Existence solutions for boundary value problem of nonlinear fractional q-difference equations," Advances in Difference Equations, vol. 2013, article 113, 2013.

[15] W. Yang, "Positive solutions for nonlinear semipositone fractional q-difference system with coupled integral boundary conditions," Applied Mathematics and Computation, vol. 244, pp. 702-725, 2014.

[16] A. Alsaedi, B. Ahmad, and H. Al-Hutami, "A study of nonlinear fractional $q$-difference equations with nonlocal integral boundary conditions," Abstract and Applied Analysis, vol. 2013, Article ID 410505, pp. 1-8, 2013.

[17] Y. Zhao, G. Ye, and H. Chen, "Multiple positive solutions of a singular semipositone integral boundary value problem for fractional $q$-derivatives equation," Abstract and Applied Analysis, vol. 2013, Article ID 643571, pp. 1-12, 2013.

[18] Y. Zhao, H. Chen, and Q. Zhang, "Existence results for fractional $q$-difference equations with nonlocal q-integral boundary conditions," Advances in Difference Equations, vol. 2013, article 48, 2013.

[19] Q. Zhao and W. Yang, "Positive solutions for singular coupled integral boundary value problems of nonlinear higher-order fractional q-difference equations," Advances in Difference Equations, vol. 2015, article 290, 2015.

[20] W. Yang and Y. Qin, "Positive solutions for nonlinear caputo type fractional $q$-difference equations with integral boundary conditions," Mathematics, vol. 4, no. 4, article 63, 2016.

[21] S. Zhang, "Positive solutions for boundary-value problems of nonlinear fractional differential equations," Electronic Journal of Differential Equations, vol. 36, pp. 1-12, 2006. 
[22] Y. Zhao, S. Sun, Z. Han, and Q. Li, “The existence of multiple positive solutions for boundary value problems of nonlinear fractional differential equations," Communications in Nonlinear Science and Numerical Simulation, vol. 16, no. 4, pp. 2086-2097, 2011.

[23] Z. Bai and H. Lü, "Positive solutions for boundary value problem of nonlinear fractional differential equation," Journal of Mathematical Analysis and Applications, vol. 311, no. 2, pp. 495-505, 2005.

[24] D. J. Guo, Nonlinear Functional Analysis, Shandong Sci. Tec. Press, Shandong, China, 2nd edition, 2001. 


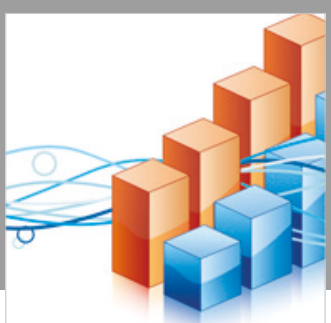

Advances in

Operations Research

\section{-n-m}
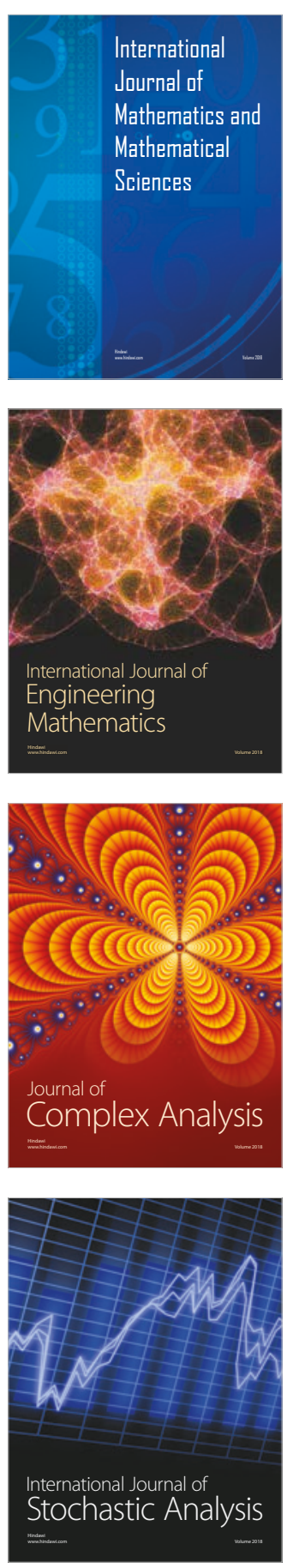
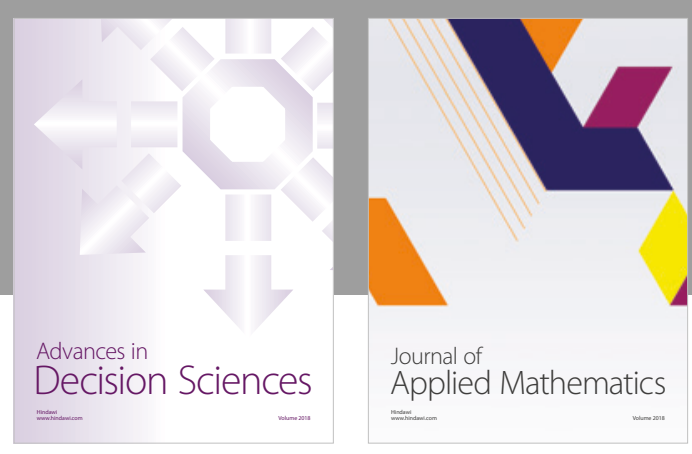

Journal of

Applied Mathematics
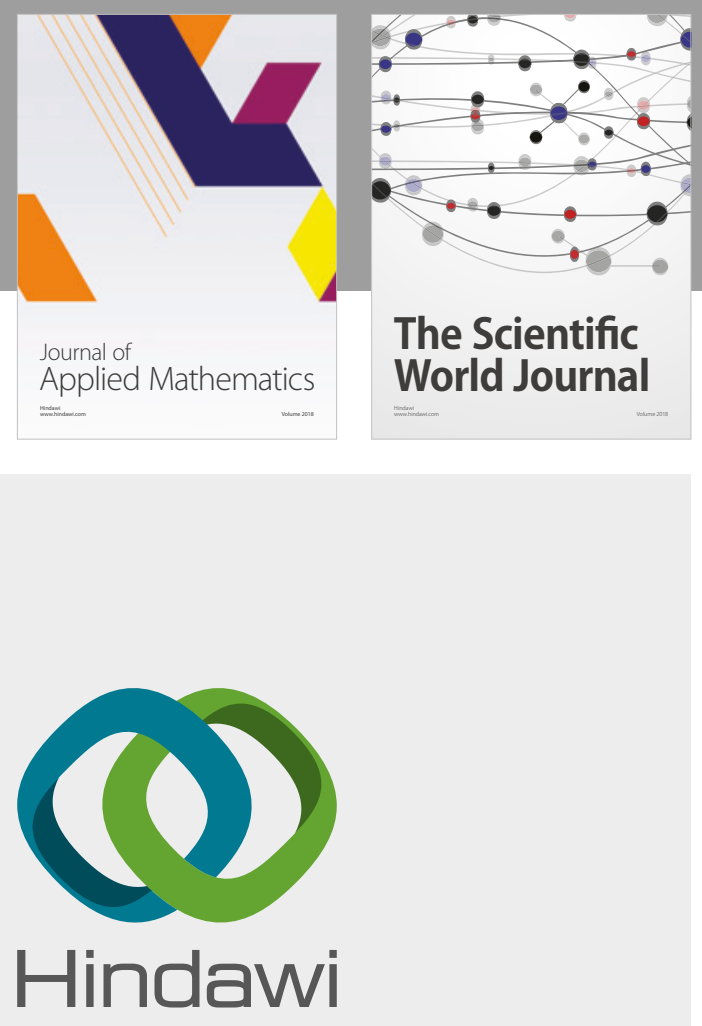

Submit your manuscripts at

www.hindawi.com

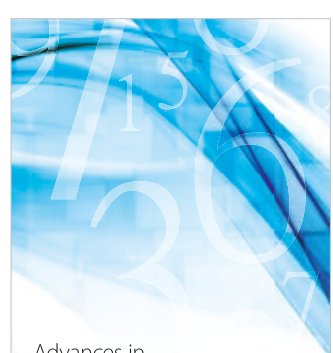

Advances in
Numerical Analysis
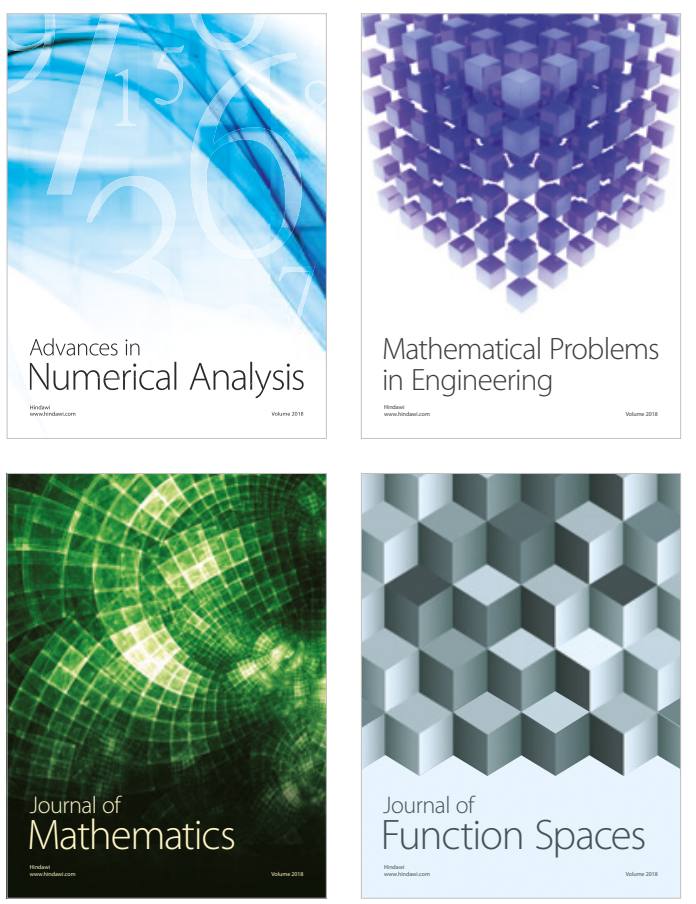

Mathematical Problems in Engineering

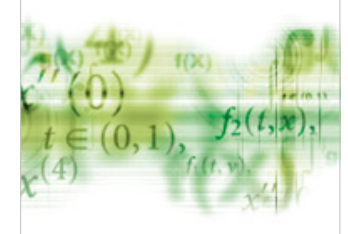

International Journal of

Differential Equations

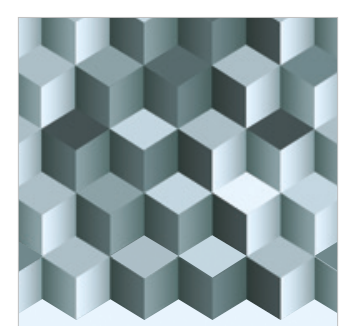

Journal of

Function Spaces

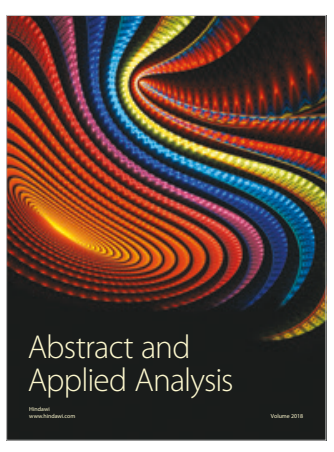

The Scientific

World Journal

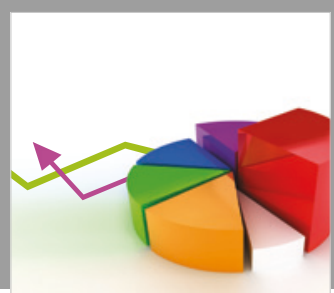

Journal of

Probability and Statistics
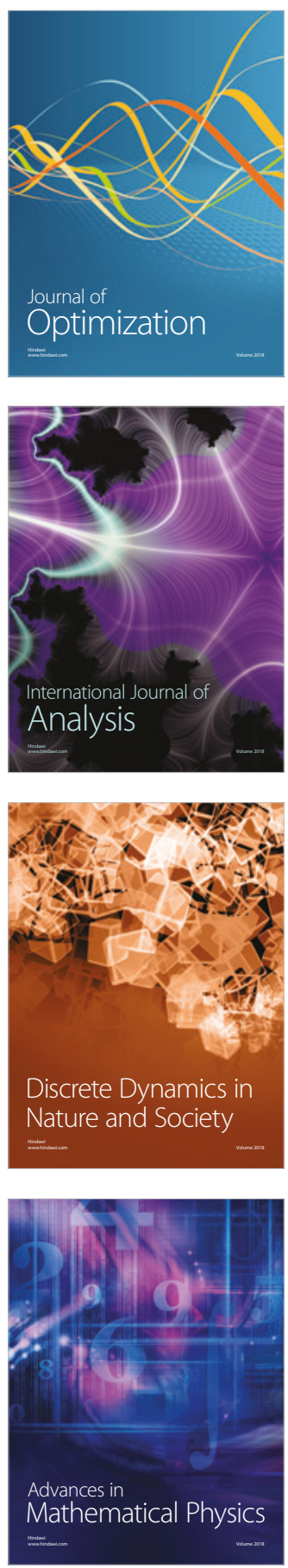